\title{
Resident-Reported Impact of a Novel Oncology Curriculum for Internal Medicine Residents
}

\author{
Frederick D. Tsai ${ }^{1,2} \cdot$ Brendan Guercio $^{3} \cdot$ Sherri Stuver $^{1,4} \cdot$ Robert Stern $^{1,2} \cdot$ Michael J. Peluso $^{5} \cdot$ Marissa Winkler $^{6}$. \\ Sorbarikor Piawah ${ }^{5}$. Mounica Vallurupalli ${ }^{1,2}$. Marlise R. Luskin ${ }^{1,2}$. David Braun ${ }^{1,2}$. Alexander Parent ${ }^{1,2}$. \\ Brett Glotzbecker ${ }^{7} \cdot$ Kerry Laing Kilbridge $\mathrm{e}^{1,2,8}$
}

Accepted: 9 June 2021 / Published online: 4 September 2021

(c) American Association for Cancer Education 2021

\begin{abstract}
The Accreditation Council of Graduate Medical Education mandates that all internal medicine residents gain exposure to internal medicine subspecialties including hematology and oncology. While many residents meet this criterion through inpatient oncology rotations, the current structure of many inpatient oncology rotations leaves little opportunity for formal education. We therefore designed a novel oncology curriculum consisting of one-page oncology teaching sheets to increase the number, breadth, and quality of formal teaching sessions on our resident inpatient oncology services. In order to evaluate the curriculum, we conducted pre- and post-intervention surveys of residents. From these surveys, we found that $72.2 \%$ of residents used the teaching sheets on their inpatient oncology rotation and that the teaching sheets led to an increase in the number of formal oncology teaching sessions (mean $3.4 \pm 2.1$ post-implementation vs $2.6 \pm 2.0$ pre-implementation, $\mathrm{p}=0.008$ ), the breadth of oncology topics taught (\% reporting $\geq 5$ topics; $26.1 \%$ vs $16.3 \%, \mathrm{p}=0.035$ ), the proportion of residents reporting improvement in overall oncology knowledge $(80.2 \%$ vs $62.4 \%, \mathrm{p}=0.012)$, and the proportion of residents reporting improvement in their ability to care for patients $(70.8 \%$ vs $48.9 \%, \mathrm{p}=0.013)$. These results demonstrate that formal oncology teaching can be improved on inpatient oncology rotations through a simple and easily replicable oncology curriculum.
\end{abstract}

Keywords Medical oncology $\cdot$ Oncology curriculum $\cdot$ Evaluation $\cdot$ Internal medicine residency training

\section{Introduction}

The Accreditation Council of Graduate Medical Education (ACGME) mandates that all internal medicine residents gain both inpatient and outpatient exposure to the spectrum of

Frederick D. Tsai and Brendan Guercio contributed equally to this work.

Brett Glotzbecker and Kerry Laing Kilbridge contributed equally to this work.

Kerry Laing Kilbridge

Kerry_Kilbridge@dfci.harvard.edu

Dana-Farber Cancer Institute, Boston, MA 02215, USA

2 Brigham \& Women's Hospital, Boston, MA 02115, USA

3 Memorial Sloan Kettering Cancer Center, New York, NY 10065, USA

4 Boston University School of Public Health, Boston University, Boston, MA 02118, USA clinical disorders seen in the internal medicine subspecialties. This includes both hematology and oncology. To meet this ACGME mandate, many internal medicine residents rotate through inpatient medical oncology services. Unfortunately, rotations through inpatient medical oncology services do not appear to increase resident interest in the field of medical oncology[1]. Our anecdotal experience is that this finding is at least partially explained by the limited time available for formal teaching on inpatient medical rotations. As demonstrated in the iCOMPARE study, which evaluated

5 University of California San Francisco Medical Center, San Francisco, CA 94143, USA

6 Massachusetts General Hospital, Boston, MA 02114, USA

7 University Hospital Seidman Cancer Center, Cleveland, OH 44106, USA

8 Lank Center for Genitourinary Oncology, Dana-Farber Cancer Institute, 450 Brookline Ave, Boston, MA 02215, USA 
a flexible duty hour schedule for internal medicine residents, residents on inpatient medical services spend only $7.3 \%$ of their time involved in educational activities [2]. Conversely, the majority of their time is spent on indirect patient care tasks. These tasks have become increasingly burdensome as a result of the growing patient censuses on resident services. At our institutions (Brigham \& Women's Hospital and DanaFarber Cancer Institute), the combined inpatient oncology census increased by approximately 20\% from 2015 to 2018 (Brigham \& Women's Hospital Oncology Services Census Data, October 2015 to June 2018, unpublished). As a result, medicine housestaff can finish their oncology rotations feeling comfortable managing the acute medical complications that arise in their patients in the hospital, but with less formal education about their underlying malignancies.

When internal medicine residents are given the opportunity to engage in formal education, their interest in the field increases [3]. With this in mind, an oncology committee within the Brigham and Women's Hospital Internal Medicine Residency Program developed a novel oncology curriculum to aid in formal teaching of the inpatient oncology service. The goals of the oncology curriculum were threefold: (1) to increase the number of teaching sessions during any given 2-week oncology rotation, (2) to increase the breadth of topics discussed, and (3) to encourage and improve teaching in a team-based setting.

\section{Methods}

At our institutions, housestaff rotate through inpatient solid tumor oncology, malignant hematology, and bone marrow transplantation services. Each service is staffed by two interns, one resident, and one attending oncologist. The residents serve as the first point of contact for admissions, triage, and emergent medical management, while the interns operate as the responding clinicians for patients admitted to these services. Each member of the housestaff completes approximately 4-6 weeks of inpatient oncology rotations per year.

The oncology curriculum was developed as a pilot program and implemented in stages over 2015-2016 academic year. To create the curriculum, a committee of junior and senior residents, one chief medical resident, and a subset of oncology attendings from the Dana-Farber Cancer Institute were convened. After discussing possible formats for the curriculum, the committee decided on succinct one-page teaching sheets that would focus on each of the major cancer types encountered by residents. The teaching sheets would include the presentation, diagnosis, staging, treatment, and prognosis for each of the common cancer types and would be written at the level of expertise of a medical resident (see Supplementary Material for an example-melanoma and renal cell carcinoma). The primary goal was for both residents and attendings on the inpatient oncology services to use the sheets as references for formal teaching sessions. With the sheets readily available, residents and attendings would not need to spend valuable time creating teaching materials on their own, thus decreasing the barrier to formal teaching sessions.

Once the format of the curriculum was finalized, junior and senior residents designed and wrote the collection of teaching sheets, working with faculty experts to ensure accuracy and applicability of content. The initial topics chosen for the teaching sheets were as follows: acute myeloid leukemia, acute promyelocytic leukemia, acute lymphoblastic leukemia, chronic lymphocytic leukemia, Hodgkin's lymphoma, non-Hodgkin lymphoma, multiple myeloma, bone marrow transplantation, breast cancer, colorectal cancer, lung cancer, prostate cancer, melanoma, and pancytopenia. Of note, since 2015-2016, the curriculum has expanded to include additional topics including: chimeric antigen receptor (CAR-T) T cells, renal cell carcinoma, pancreatic cancer, stem cell of origin in myeloid and lymphoid malignancies, pulmonary complications of bone marrow transplant, hepatic complications of bone marrow transplant, and graft versus host disease.

Once the first full set of teaching sheets were completed, they were made available to all attendings and housestaff on a residency intranet site. Prior to their inpatient oncology rotations, housestaff and attendings received an email notification outlining the goals of the oncology curriculum and setting forth the expectation that each inpatient oncology team has regular formal teaching sessions led by attendings and upper level residents. The exact number and format of these sessions were left to the discretion of each individual team.

In order to evaluate the oncology curriculum, both preand post-intervention surveys of the residents were completed. The pre-intervention surveys were completed by residents who rotated on an inpatient oncology service in the five months prior to roll-out of the curriculum from midAugust 2015 to mid-January 2016. The post-intervention surveys were completed by residents who rotated on an inpatient oncology service in 5 months after implementation of the curriculum late January 2016 through late June 2016. These surveys assessed the number of teaching sessions, the number of topics covered, and the overall quality of teaching on the resident oncology services. The post-intervention surveys also examined use and uptake of the teaching sheets, overall satisfaction with oncology learning, and the curriculum's impact on patient care. The results were anonymized and analyzed in aggregate at the end of the study period. For continuous variables, comparisons between pre- and post-intervention mean values were assessed using t-tests. For categorical variables, comparisons between pre- and 
post-intervention frequencies were assessed using Chisquare tests; if any cell counts were less than five, then Fisher's exact tests were used. All analyses were performed in SAS 9.4 (Cary, NC). The survey evaluation of the oncology curriculum was submitted to the Partners Institutional Review Board (IRB) for review and was deemed to be exempt.

\section{Results}

\section{Study Population Characteristics}

Prior to implementation of the oncology curriculum, 180 housestaff were invited to complete a pre-intervention survey, of which 98 (54\%) responded. Following implementation of the oncology curriculum, 216 housestaff were invited to complete a post-intervention survey, of which 115 (53\%) responded. Six additional survey responses in the pre-intervention group and four in the post-intervention group were excluded from the analyses because they only included one completed answer. Incomplete responses to individual survey questions were not included in the question's response denominator. Descriptive statistics comparing individuals who completed the pre-intervention survey to those who completed the post-intervention survey are displayed in Table 1. There was no significant difference between the pre-intervention and post-intervention groups in terms of oncology rotation type, age, gender, number of oncology rotations completed during residency, interest in the field of hematology/oncology, or the proportion of respondents who were interns versus residents $(p>0.05)$.

\section{Comparison of Pre- and Post-intervention Survey Responses}

Of the 115 post-intervention respondents, $72.2 \%(83 / 115)$ reported using the oncology teaching sheets during their inpatient oncology rotations. After the oncology curriculum was introduced, residents noted a statistically significant increase in the number of formal teaching sessions during a rotation (mean $2.6 \pm 2.0$ standard deviation pre-intervention vs mean $3.4 \pm 2.1$ standard deviation post-intervention, $p=0.008$; Fig. 1a). Residents also reported a significant increase in the number of oncology topics covered during a rotation, $16.3 \%$ (16/98) covered 5 or more topics pre-intervention vs $26.1 \%(30 / 115)$ covered 5 or more topics postintervention ( $p=0.035$; Fig. 1b). With regard to the educational benefit derived from the curriculum, $80.2 \%(85 / 106)$ of responding housestaff agreed or strongly agreed that formal teaching sessions during their rotation had improved their overall knowledge of oncology, compared to only $62.4 \%(58 / 93)$ of housestaff responding pre-intervention $(p=0.012$; Fig. 2a). Similarly, $70.8 \%$ (75/106) of respondents post-intervention agreed or strongly agreed that formal teaching sessions improved their ability to care for their patients compared to only $48.9 \%$ (46/94) of respondents pre-intervention ( $p=0.013$; Fig. $2 b)$.
Table 1 Participant characteristics

\begin{tabular}{lll}
\hline & $\begin{array}{l}\text { Pre-intervention } \\
(\mathrm{N}=98)\end{array}$ & $\begin{array}{l}\text { Post-intervention } \\
(\mathrm{N}=115)\end{array}$ \\
\hline Most recent oncology service rotated on, $\mathrm{n}(\%)$ & & \\
Solid tumor oncology & $24(24.5 \%)$ & $21(18.3 \%)$ \\
Malignant hematology team 1 & $17(17.4 \%)$ & $20(17.4 \%)$ \\
Malignant hematology team 2 & $14(14.3 \%)$ & $21(18.3 \%)$ \\
Oncology night float & $29(29.6 \%)$ & $28(24.4 \%)$ \\
Bone marrow transplant & $14(14.3 \%)$ & $25(21.7 \%)$ \\
Intern & $63(64.3 \%)$ & $70(60.9 \%)$ \\
Resident & $35(35.7 \%)$ & $45(39.1 \%)$ \\
Age - mean \pm SD (years) & $29.2 \pm 2.4$ & $29.9 \pm 2.8$ \\
& {$[\mathrm{~N}=93]$} & {$[\mathrm{N}=102]$} \\
Gender, $\mathrm{n}(\%)$ & {$[\mathrm{N}=93]$} & {$[\mathrm{N}=102]$} \\
Female & $37(39.8 \%)$ & $39(38.2 \%)$ \\
Male & $56(60.2 \%)$ & $63(61.8 \%)$ \\
Number of oncology rotations completed, mean $\pm \mathrm{SD}$ & $2.4 \pm 1.3$ & $2.6 \pm 1.3$ \\
& {$[\mathrm{~N}=93]$} & {$[\mathrm{N}=102]$} \\
Indicated hematology/oncology as their field of interest, $\mathrm{n}(\%)$ & $37 / 94(39.4 \%)$ & $30 / 102(29.4 \%)$ \\
\hline
\end{tabular}

Descriptive characteristics of housestaff comparing pre-oncology curriculum responders to post-oncology curriculum responders. Six individuals from the pre-survey group and four from the post-survey group were not included in the analyses, because they only responded to one question regarding the overall quantity of teaching

Abbreviation: $S D$ standard deviation 
Fig. 1 a Frequency histogram comparing trainee-reported number of formal teaching sessions on oncology rotations preand post-implementation of the oncology curriculum teaching sheets. b Frequency histogram comparing trainee-reported number of oncology topics covered in formal oncology teaching sessions during oncology rotations pre- and postimplementation of the oncology curriculum teaching sheets a

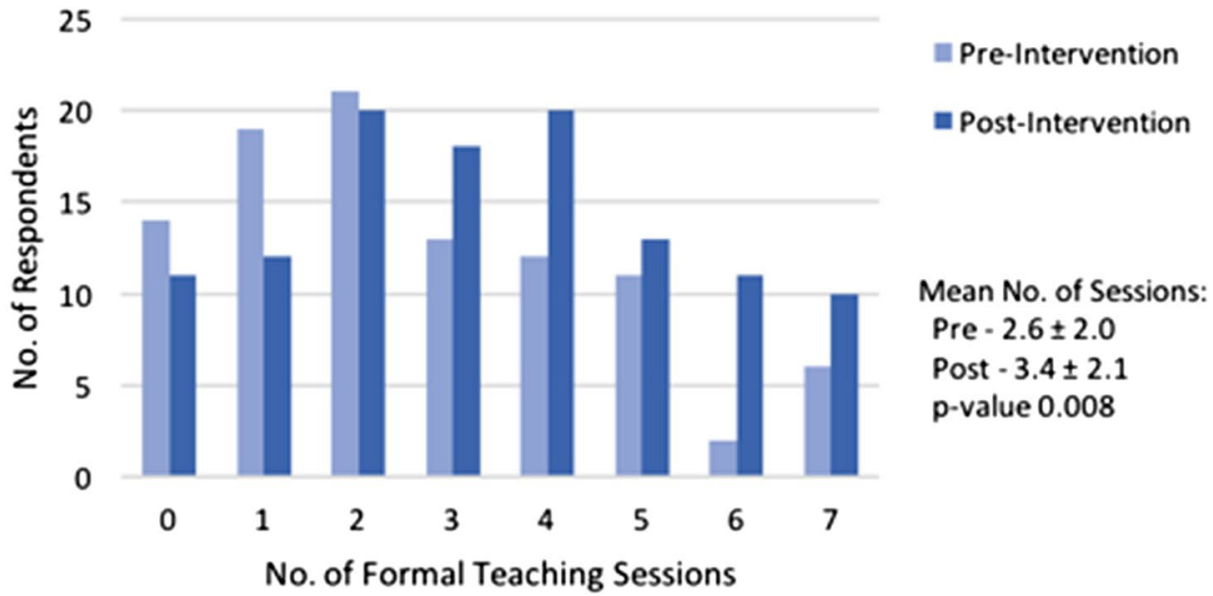

b

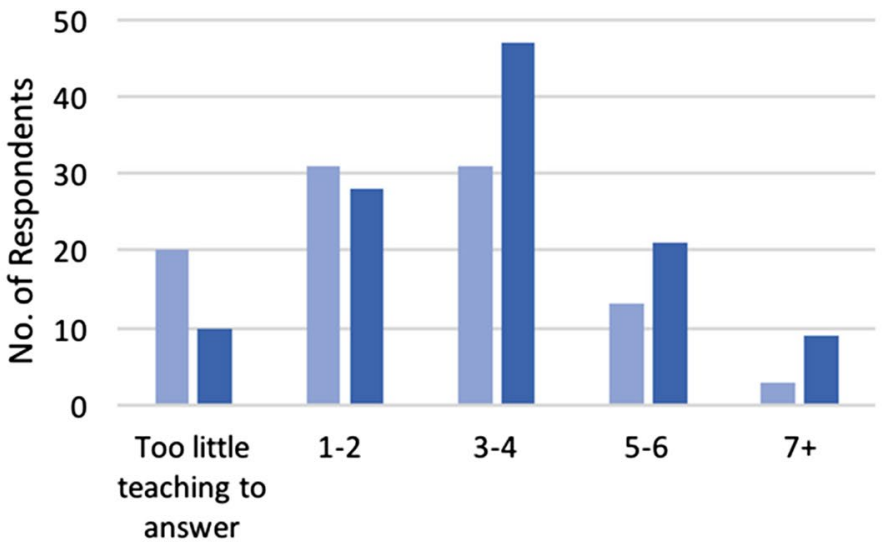

Pre-Intervention

- Post-Intervention p-value 0.035

No. of Topics Covered

\section{Qualitative Comments on Impact of the Oncology Curriculum}

Besides the quantitative responses, the post-intervention survey allowed respondents to write free text comments regarding their impressions of the oncology curriculum teaching sheets. These qualitative comments highlighted the curriculum's ease of use, relevance, and versatility. For example:

[The oncology curriculum teaching sheets [f]ocused on topics relevant to current active patients... and seemed to be [at] a good level for me as a resident teacher and the interns [and] med student. I learned the topics I taught about in much greater depth and am retaining the information more easily. I was able to try a few different teaching approaches - discussion, chalk talk, asking questions versus presenting information. It was a good reminder to teach more and tackle short topics - including those beyond the topics included in the onc curriculum.

The [oncology teaching] sheets are a tremendous reference material for sitting down one on-one with the intern.

Such comments also noted unintended benefits of the curriculum, such as facilitation of independent, individual learning:
[The curriculum was] crucial for my independent personal learning and... incredibly high yield.

There isn't time for adequate teaching on the service because you are so busy with patient care. I really like the sheets, because I can use them individually.

Several faculty involved in creating the oncology curriculum teaching sheets with residents submitted unsolicited commentary (collected separately from the formal surveys) that demonstrated the curriculum was also valued by faculty and that the project promoted relationships between faculty and residents:

It was a pleasure working with , she did a great job with this!

This is a major contribution to our residency.

\section{Discussion}

Through the creation of an oncology curriculum consisting of succinct, one-page oncology teaching sheets, we aimed to improve the formal educational teaching on our inpatient oncology rotations. We found that implementation 
Fig. 2 a Frequency histogram comparing trainee-reported improvement in general oncology knowledge by formal teaching sessions pre- and postimplementation of the oncology curriculum teaching sheets. b Frequency histogram comparing trainee-reported improvement in the ability to care for patients pre- and post-implementation of the oncology curriculum teaching sheets a

80

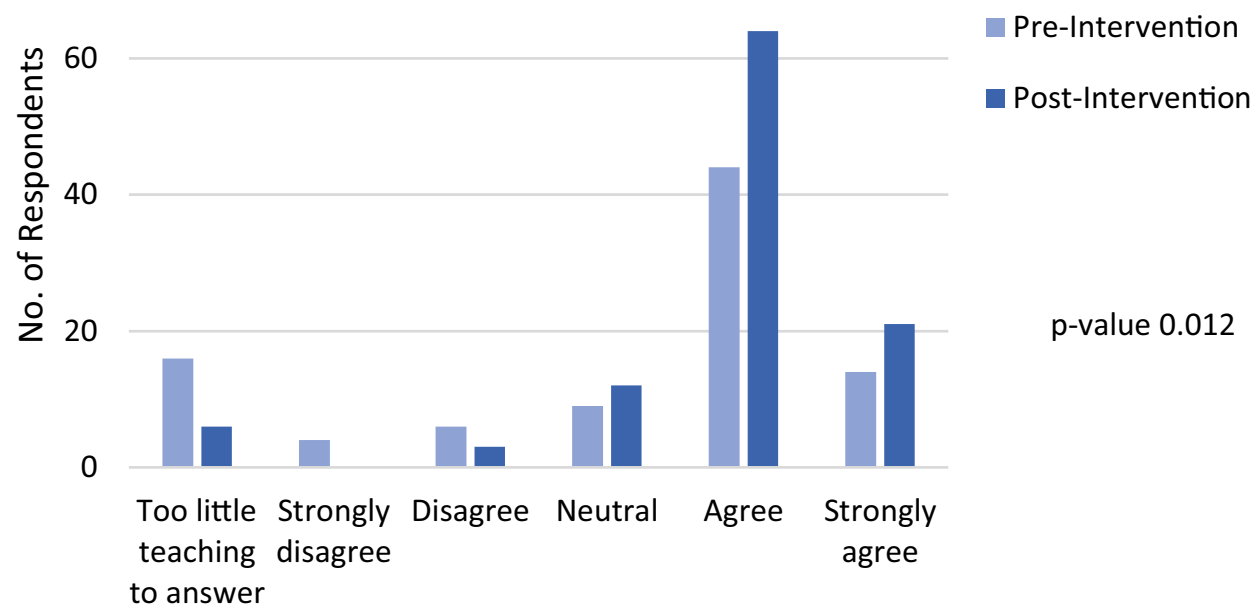

b

80

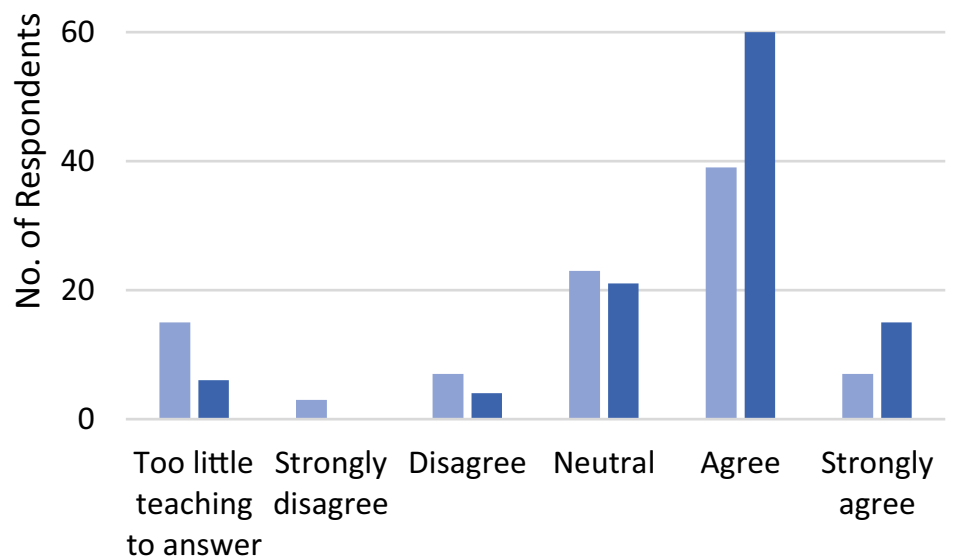

Pre-Intervention

Post-Intervention

p-value 0.013 of the curriculum was associated with statistically significant improvements in the quantity and breadth of oncology teaching and led to self-reported improvements in oncology knowledge and the ability to care for oncology patients.

Existing literature suggests that the development of core educational curricula are essential for internal medicine resident training [4]. However, the current internal medicine resident training environment leaves little room for such formal education. Duty hour restrictions, indirect patient care responsibilities, and rising patient censuses prevent residents and attendings from preparing and conducting formal educational sessions $[2,4]$. To cope with such challenges, novel educational tools focused on efficient learning, spaced learning, and right-timed learning are required. Our curriculum was designed to meet these goals. By creating readily available, high yield lesson plans in the form of teaching sheets, we intended to significantly reduce the time required of residents to create a teaching plan for interns on their team.

The need for oncology-specific education within internal medicine residency training is also increasing. Primary care physicians and other internal medicine specialists play an increasingly important role in the care of oncology patients and cancer survivors following their primary oncology treatment [5]. Yet oncology teaching tools are lacking or insufficient at many North American training centers [6, 7]. This is despite evidence demonstrating that implementation of such tools improves resident interest and knowledge in medical oncology and its related fields [8, 9]. In a study with results like our own, Lycan et al. randomized 29 internal medicine residents to receive either a traditional American Board of Internal Medicine oncology review lecture focused on management of acute complications in cancer patients or 
an oncology-specific lecture reviewing landmark studies in oncology treatment and prognostication. Similar to our findings that residents judged the oncology-specific curriculum to improve their ability to care for patients, the investigators showed that resident exposure to the landmark studies lecture resulted in superior prognostication and less inappropriate withdrawal of care when residents were queried in post-lecture clinical vignettes [3].

Moving forward, it will be necessary to determine whether our oncology curriculum led to objective changes in resident knowledge of oncology and improvement in the care of oncology patients. The former could be assessed through Internal Medicine In-Training Examination results, while the latter could be determined through objective measures of patient outcomes. In addition, it will be crucial to explore the effect of the curriculum on competency-based resident education in internal medicine [10]. Given the success of the oncology curriculum, we have continued to update the teaching sheets with the goal of revisiting content annually with faculty experts. We are in the midst of the 2020-2021 update of our current teaching sheets with an expansion of the curriculum to new topics, such as immunotherapies and their complications, the impact of the COVID-19 pandemic on treatment decisions, and an overview of bone marrow conditioning regimens. A survey reassessment of the curriculum is planned for the current academic year, 2020-2021.

Our study had several important limitations. First, the survey response rate in our study was only $53-54 \%$. However, this rate of response is comparable to similar studies in the literature $[5-7,11,12]$. Second, though our study captured self-perceived resident competence and knowledge in oncology before and after implementation of our oncology curriculum, we did not collect objective measures of these parameters. Third, our results could be confounded by the natural maturation of residents and interns over the course of the training year in which implementation of the oncology curriculum took place. Fourth, it is possible that the residents who participated in the survey bias the results in favor of the oncology curriculum. Because we do not have data for the non-responding residents, we cannot quantify the extent to which possible participation bias may affect results.

In sum, a resident-designed oncology curriculum resulted in increased quantity and quality of formal oncology teaching on inpatient oncology rotations. We believe that such a curriculum could be easily expanded both within our own residency program and to additional residency programs that are interested in improving inpatient oncology education. Lastly, we expect that these teaching sheets will be valuable tools for attendings as well as residents given the increased outpatient subspecialization of academic oncologists and the challenge that this poses for teaching on a general inpatient oncology service.
Supplementary Information The online version contains supplementary material available at https://doi.org/10.1007/s13187-021-02055-6.

Acknowledgements This project was made possible with the generous support of the Brigham and Women's Hospital Department of Medicine Macy Scholarship Award, Ted Alyea, MD PhD, Joseph O. Jacobson MD MSc, and Craig A. Bunnell, MD, MPH, MBA. Thank you to Amin Nassar MD for the most recent updates to the renal cell carcinoma teaching sheet.

Author Contribution All authors contributed to the study conception, material preparation, and design. Data collection and analysis were performed by Michael Peluso, Sherri Stuver, Brett Glotzbecker, and Kerry Kilbridge. The first draft of the manuscript was written by Frederick Tsai and Brendan Guercio. All authors commented on previous versions of the manuscript. All authors read and approved the final manuscript.

Data Availability Not applicable.

Code Availability Not Applicable.

\section{Declarations}

Conflict of Interest The authors declare no competing interests.

\section{References}

1. McFarland DC, Holland J, Holcombe RF (2015) Inpatient hematology-oncology rotation is associated with a decreased interest in pursuing an oncology career among internal medicine residents. J Oncol Pract 11(4):289-295. https://doi.org/10.1200/jop.2015. 003798

2. Desai SV, Asch DA, Bellini LM, Chaiyachati KH, Liu M, Sternberg AL, Tonascia J et al (2018) Education outcomes in a duty-hour flexibility trial in internal medicine. N Engl J Med 378(16):1494-1508. https://doi.org/10.1056/NEJMoa1800965

3. Lycan T, Hansen K, Miller D (2017) Impact of curriculum content on resident attitudes towards cancer care. J Cancer Educ 32(2):280-282. https://doi.org/10.1007/s13187-015-0908-y

4. Di Francesco L, Pistoria MJ, Auerbach AD, Nardino RJ, Holmboe ES (2005) Internal medicine training in the inpatient setting. A review of published educational interventions. J Gen Intern Med 20(12):1173-1180. https://doi.org/10.1111/j.1525-1497.2005. 00250.x.

5. Susanibar S, Thrush CR, Khatri N, Hutchins LF (2014) Cancer survivorship training: a pilot study examining the educational gap in primary care medicine residency programs. J Cancer Surviv 8(4):565-570. https://doi.org/10.1007/s11764-014-0366-2

6. Cheung WY, Fishman PN, Verma S (2009) Oncology education in Canadian undergraduate and postgraduate training programs. J Cancer Educ 24(4):284-290. https://doi.org/10.1080/0885819090 2973143

7. Tam VC, Berry S, Hsu T, North S, Neville A, Chan K, Verma S (2014) Oncology education in Canadian undergraduate and postgraduate medical programs: a survey of educators and learners. Curr Oncol 21(1):e75-88. https://doi.org/10.3747/co.21.1667

8. Mulder SF, Bleijenberg G, Verhagen SC, Stuyt PM, Schijven MP, Tack CJ (2009) Improved competence after a palliative care course for internal medicine residents. Palliat Med 23(4):360-368. https://doi.org/10.1177/0269216308100250 
9. Sloan DA, Plymale MA, Donnelly MB, Schwartz RW, Edwards MJ, Bland KI (2004) Enhancing the clinical skills of surgical residents through structured cancer education. Ann Surg 239(4):561-566. https://doi.org/10.1097/01.sla.0000118568. 75888.04

10. Green ML, Aagaard EM, Caverzagie KJ, Chick DA, Holmboe E, Kane G, Smith CD, Iobst W (2009) Charting the road to competence: developmental milestones for internal medicine residency training. J Grad Med Educ 1(1):5-20. https://doi.org/10.4300/01. 01.0003

11. Chen H, Mortazavi A, Levine E, Kamisetti S, Kamisetti S, Ramnath N (2007) What did you think of the hematology/oncology rotation? A survey of internal medicine residents. J Cancer Educ 22(1):50-55. https://doi.org/10.1007/bf03174376

12. Taylor RE, Macdonald R (2000) Education and training of senior house officers in clinical oncology departments. Clin Oncol ( $R$ Coll Radiol) 12(1):42-47. https://doi.org/10.1053/clon.2000.9109

Publisher's Note Springer Nature remains neutral with regard to jurisdictional claims in published maps and institutional affiliations. 